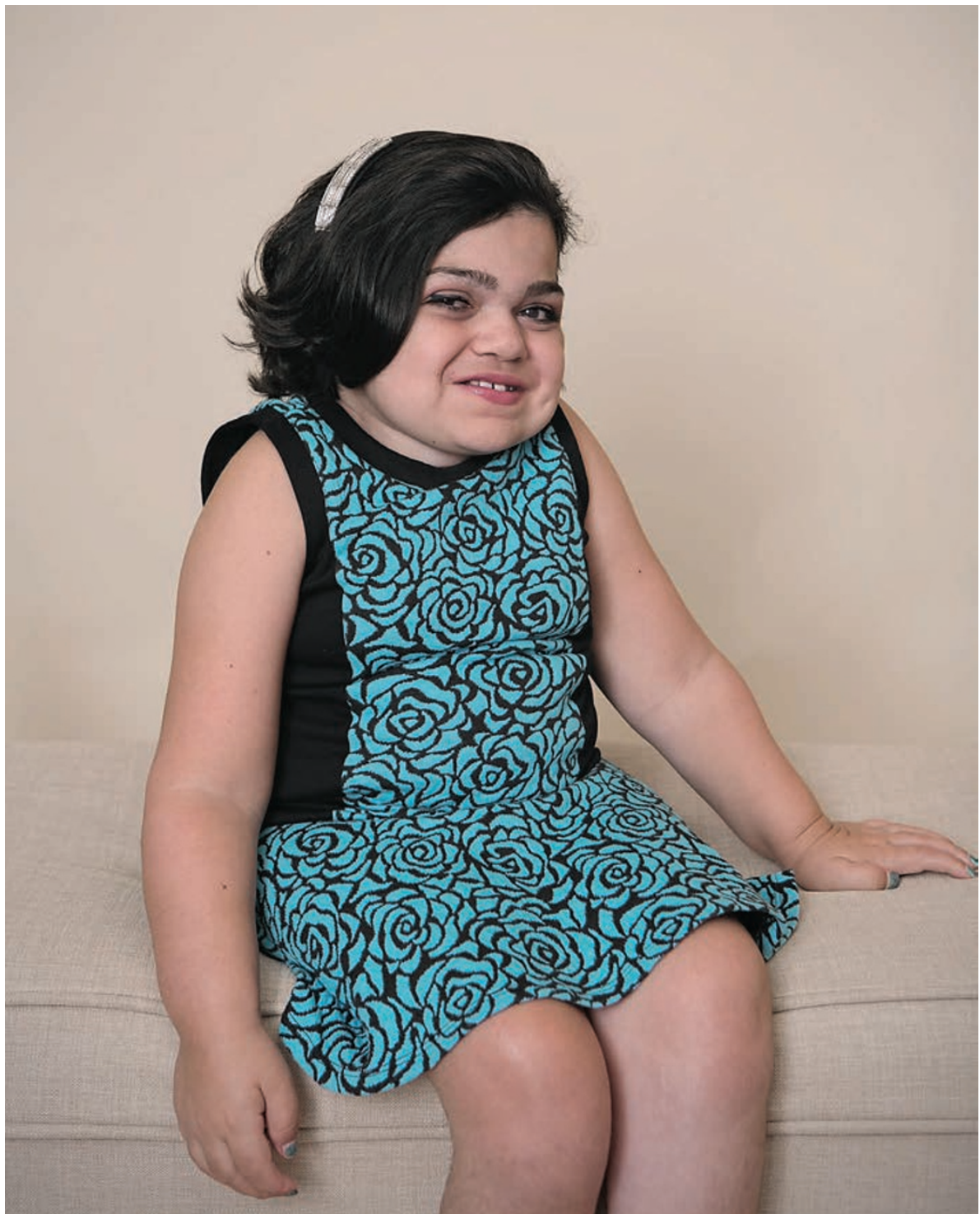

The outlook for children like Michelle Hopkins may improve if efforts to use gene therapy are successful.

\title{
GENE THERAPY
}

\section{A new chapter}

\section{In the 1980s, bone-marrow transplants transformed the lives of children with fatal lysosomal diseases. Researchers are now working on another revolutionary treatment.}

\section{BY ANTHONY KING}

$\mathrm{T}$ hree years ago, 33-year-old Paul Franklin died from sudden heart failure. In some ways he was an average young man. Despite being wheelchair-bound, he worked on Fridays in his local McDonalds in Croydon, south London. He enjoyed going to the cinema and attending football matches. "He was always optimistic and happy, and had lots of friends," name of gargoylism. It is caused by a fault in the gene that produces an enzyme known as a-L-iduronidase (IDUA). Untreated children suffer from cognitive decline, heart and liver abnormalities, deafness and bone problems.

The pioneer of bone-marrow transplants for Hurler syndrome was pathologist John Hobbs at Westminster Children's Hospital in London. Hobbs had performed bone-marrow transplants for leukaemia, but Paul's was the first for any LSD. Transplants involve using chemotherapy to knock out the child's own stem cells, and implanting white blood cells from a donor who has functioning IDUA.

Early transplants for Hurler patients were risky - about 50\% died - but life without a transplant was unpleasant. Paul's parents had met a child with Hurler syndrome who had a painful life before dying at the age of seven. "We knew what the prognosis was," says Anne. "Still, it was the hardest decision we had ever made." For Paul, the gamble paid off. The procedure transformed his life, as it did for subsequent patients.

These days, the chances of surviving a transplant are high, up to $95 \%$, but it is not a cure. Even children who have the procedure can have problems with bone development, says Chester Whitley, a paediatrician at the University of Minnesota in Minneapolis, who performed the first bone-marrow transplant for Hurler syndrome in North America. "They are dwarfed and, if we don't get to them early enough, they can suffer significant brain damage."

Early transplantation helps, but cognitive function is not always preserved. Paul was fortunate: he needed only infrequent hospital visits. The next transplant recipient after Paul lived into her mid-30s, although her cognitive age was only around seven.

Even after a transplant, it is extremely rare for a person with Hurler syndrome to be able to live independently; most need regular medical care. And now that several US states have begun screening newborns for the syndrome, pressure is mounting for better treatment.

"We are looking towards a treatment that embraces as many systems as possible because of the multi-organ nature of the disease," says Christine Lavery, chief executive of the Society for Mucopolysaccharide Diseases in the United Kingdom. "And something that doesn't have the complication of making you a patient."

\section{ENZYME MACHINES}

Now, 36 years after the first bone-marrow transplant transformed the outlook for children with Hurler syndrome, another revolutionary treatment aims to go even further. Gene therapy promises to restore full enzyme function to people with Hurler syndrome, and then for those with other LSDs, as several approaches are either in or close to clinical trials.

The idea of gene therapy for Hurler syndrome is to replace or restore the damaged 
IDUA gene, enabling the body to produce its own enzymes. The enzymes can then leave repaired cells and enter others that are deficient in IDUA - a phenomenon known as cross-correction.

But which cells to target, and how to get the genes in, remain open questions. Viruses are by far the most popular vectors for gene therapy, and they are usually targeted at blood, liver or brain cells. One of the simplest approaches is to take the patient's haematopoietic stem cells (which give rise to blood cells) and insert the missing gene in the lab by using a lentiviral vector - a defanged HIV. This technique requires a dose of chemotherapy to suppress the remaining stem cells so that they don't replicate and swamp the repaired cells. "It's similar to what happens in a bone-marrow transplant," says geneticist Simon Jones, who pursues this strategy in his preclinical research at the Manchester Centre for Genomic Medicine, UK.

This technique has been successfully used to treat another LSD. Since 2010, the San Raffaele Telethon Institute for Gene Therapy in Milan, Italy, has used it to treat 24 children who had metachromatic leukodystrophy - a fatal disorder caused by mutations in the gene that encodes arylsulfatase A (ARSA). The team was able to halt the disease's progression, and even prevent it in some cases (M. Sessa et al. Lancet 388, 476-487; 2016). "The severe form of the disease starts in the first two years of life. But if we treat them at the very beginning, we see a lack of disease onset," says geneticist Luigi Naldini, director of the institute. "These children are healthy today." Pharmaceutical company GlaxoSmithKline has licensed the therapy, and Naldini hopes that the treatment will be available for metachromatic leukodystrophy in 2018. His group expects to start a similar trial for Hurler syndrome next year.

Whitley, meanwhile, is trying to find out whether a lentiviral vector can be delivered directly to liver cells in the body. Liver cells are a good target because they are plentiful and are natural protein factories. As a bonus, working with differentiated cells rather than stem cells means that there is no need for chemotherapy. His team has successfully delivered the IDUA gene into a mouse model of Hurler syndrome, and is now trying to scale it up and devise a more efficient delivery system. "I still have hopes," says Whitley, but he acknowledges that this is a dynamic field. "These other guys are moving so fast, and have such great ideas, that they may outstrip what we are doing."

Lentiviruses can deliver genes to both dividing and non-dividing cells, but they have a preference for targeting active genes, which can lead to harmful mutations. Another option is to deliver genes by using adeno-associated viruses (AAVs), which do not integrate genes directly into the chromosome as lentiviruses do, but instead leave the DNA floating free in the nucleus in a small-circular episome that acts as a mini-chromosome. This reduces the

risk of mutagenicity, but it makes AAVs less suitable for targeting dividing cells because the episomes do not replicate and are gradually diluted. Furthermore, although AAVs are usually well tolerated, they can elicit an immune response. "Generally, you can only give the vector once," says Whitley.

The AAVs' payload can be combined with nuclease enzymes for gene editing. This enables the gene to be spliced into the cell's DNA at a predetermined location, reducing the risk of an off-target insertion. Researchers at Sangamo BioSciences in Richmond, California, use AAVs to deliver zinc-finger nucleases and IDUA to liver cells in the patient's body.

"We only have to edit a small number of liver cells, predicted to be less than $1 \%$," says Michael Holmes, Sangamo's vice-president of research. "That will give a sufficient amount of enzyme to be therapeutic." As the liver grows, it replicates the edit, generating enzymes throughout the person's life. Sangamo is planning a phase I/II trial involving up to 12 adults with Hurler syndrome this year, and hopes to begin a trial for Hunter syndrome (MPS II) next year.

There is a potential non-viral option to use as a vector: transposons, which are DNA sequences that can insert a gene into a chromosome at random. Geneticist Perry Hackett,

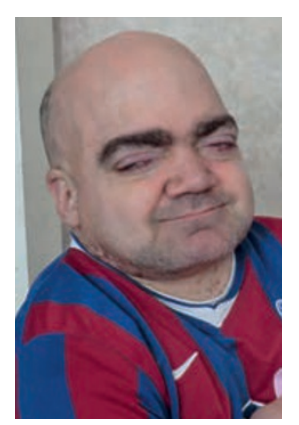

"We linew what the prognosis was. Still, it was the hardest decision we had ever made." who is also at the University of Minnesota, discovered the Sleeping Beauty transposon in 1997 (so named because he 'revived' it from an ancient form found in fish).

Hackett tested the transposon in mouse models of Hurler syndrome, delivering it to liver cells by using a massive pressure change that was "like a sonic boom", he says. He cured the IDUA deficiency in the mice, but he was unable to scale up the technique to larger animals. So he switched to cell delivery, licensing the technology to startup Immusoft, based in Seattle, Washington, which hopes to start a clinical trial next year.

In cell delivery, white blood cells are extracted from a patient with Hurler syndrome, and the Sleeping Beauty transposons transfer the IDUA gene into the cells, which are then returned to the patients. Because the transposon system is non-viral, no immunological reactions are anticipated, and transposons are also easier to produce. "DNA is a fraction of the cost of a virus, and can easily scale to treating hundreds of thousands of patients very quickly," says Matthew Scholtz, Immusoft's chief executive.
These gene therapies target body cells and not the brain cells so often compromised by LSDs, but even so, patients seem to experience cognitive benefits - although no one is quite sure how. "Liver cells, after the Sangamo gene editing, may make enough enzyme that it bombards the blood-brain barrier," says Whitley, who is a consultant for the biotech firm.

\section{DIRECT THINKING}

But instead of hoping that some enzymes will cross into the brain, another biotech firm, Regenxbio in Rockville, Maryland, is targeting the brain directly, using technology developed by medical geneticist James Wilson at the University of Pennsylvania in Philadelphia. Wilson's team has collected a menagerie of AAVs that have different properties. He explains that an AAV is the vector of choice for non-dividing cells such as neurons because the episomes last for the lifetime of the cell.

The team discovered that one particular AAV, known as AAV9, had the ability to diffuse into the brain. When it was injected directly into the cerebrospinal fluid of dog models of Hurler syndrome, signs of the disease began to disappear within a few weeks. "You get broad distribution of the vector throughout the brain and spinal cord," says Wilson.

Wilson licensed the technology to Regenxbio, which hopes to start a phase I/II trial in adults with Hurler syndrome in the United States and Canada in 2017. Wilson, who is chief scientific adviser to Regenxbio, then plans to apply the technology to Hunter syndrome. "If we can figure out how to do it in one MPS, we think it would be the same for all these conditions."

Gene-therapy trials are already forging ahead for other LSDs. In May 2013, French biotech company Lysogene completed a phase I/II study in people with Sanfilippo type A (MPS IIIA), using intracerebral injections of an AAV vector. Samantha Parker, Lysogene's chief patient access officer, says that the company plans to start a phase II/III study in 2017, using a second-generation product. In May, Abeona Therapeutics, based in Cleveland, Ohio, enrolled its first patient with MPS IIIA in a phase I/II trial, also using an AAV vector. And a clinical trial in people with a subtype of Batten disease began this year at the Nationwide Children's Hospital in Columbus, Ohio, also using AAV9. At the preclinical phase, Wilson is using gene editing with CRISPR-Cas9 to evaluate it for use in Hurler syndrome.

"No one knows the right approach yet," says Jones. "But the more approaches that are taken, the more likely we are to get something that works for patients." There is clearly an air of cautious optimism. "I hate to use the word 'cure' until the data are in," says Whitley, "but what we have is the closest I've ever seen."

Anthony King is a freelance science writer based in Dublin. 